\title{
HOMOGENIZATION IN PERFORATED DOMAINS WITH RAPIDLY PULSING PERFORATIONS
}

\author{
Doina Cioranescu ${ }^{1}$ And Andrey L. Piatnitski ${ }^{2,3}$
}

\begin{abstract}
The aim of this paper is to study a class of domains whose geometry strongly depends on time namely. More precisely, we consider parabolic equations in perforated domains with rapidly pulsing (in time) periodic perforations, with a homogeneous Neumann condition on the boundary of the holes. We study the asymptotic behavior of the solutions as the period $\varepsilon$ of the holes goes to zero. Since standard conservation laws do not hold in this model, a first difficulty is to get a priori estimates of the solutions. We obtain them in a weighted space where the weight is the principal eigenfunction of an "adjoint" periodic time-dependent eigenvalue problem. This problem is not a classical one, and its investigation is an important part of this work. Then, by using the multiple scale method, we construct the leading terms of a formal expansion (with respect to $\varepsilon$ ) of the solution and give the limit "homogenized" problem. An interesting peculiarity of the model is that, depending on the geometry of the holes, a large convection term may appear in the limit equation.
\end{abstract}

Mathematics Subject Classification. 35B27, 74Q10, 76M50.

Received May 28, 2002. Revised March 3, 2003.

\section{INTRODUCTION}

The homogenization of elliptic and parabolic problems in perforated domains is a well-developed field. There are many mathematical and numerical works devoted to the subject (see, for instance $[4-6,8,9]$ and the references herein). In all these works the geometry of the "holes" was assumed to be fixed in the time variable. In this case, the homogenization procedure for elliptic and parabolic problems is essentially the same. The averaging problems for parabolic equations with rapidly oscillating coefficients both in space and time variables, have been considered in $[3,6,10]$ or [13] for example. It was shown in $[3,10]$ and [13], that for divergence form operators, "usual" homogenization results hold. In the presence of lower order terms, new phenomena can appear. For instance, in the case of an operator with coefficients random in time, the limit problem might remain random (see [11] and [13]).

The aim of this paper is to study a class of domains whose geometry strongly depends on time. Namely, we consider here a model which involves a microstructure pulsing rapidly in time. Consequently, the corresponding parabolic operator will be defined in a perforated domain with rapidly pulsing periodic perforations. We assume

\footnotetext{
Keywords and phrases. Homogenization, perforated domains, pulsing perforations, multiple scale method.

1 Laboratoire Jacques-Louis Lions (Analyse Numérique), Université Paris VI - CNRS, 175 rue du Chevaleret, 75013 Paris, France; e-mail: cioran@ann.jussieu.fr

2 Narvik University College HiN, Department of Mathematics, P.O. Box 385, 8505 Narvik, Norway.

3 Lebedev Physical Institute, Russian Academy of Science, Leninski Prospect 53, Moscow 117333, Russia;

e-mail: andrey@sci.lebedev.ru
} 
that at the boundary of the holes homogeneous Neumann condition in the space variables is stated, while the enveloping domain usually coincides with $\mathbb{R}^{n}$.

Let us point out that since standard conservation laws do not hold in the model, even getting a priori estimates leads to certain difficulties. We show that energy type estimates can be obtained in a weighted space where the weight is the principal eigenfunction of an "adjoint" periodic time-dependent eigenvalue problem. This problem is not a classical one, and its investigation is an important part of this work. Then the multiple scale method $(c f .[1,2])$ is used in order to construct the leading terms of a formal expansion of the solution, whose justification relies on the "weighted" energy estimates mentioned above.

One interesting peculiarity of the model under consideration is the appearance of a large convection term in the limit equation. This means that in general, the limit diffusive dynamics can be observed only in moving spatial coordinates and the corresponding velocity grows like negative power of the microscopic length scale.

The technique also applies to problems in bounded perforated domains with Dirichlet boundary condition on the exterior boundary while the Neumann exterior condition requires more delicate treatment. This will be done in a forthcoming work.

The paper is organized as follows. In the first section we introduce necessary notation and pose the problem. Section 2 deals with auxiliary eigenvalue problems. We are interested in the behaviour of the ground state. Section 3 is devoted to a priori energy estimates. Then, in the next two sections (4 and 5), we construct and justify the asymptotic expansion of the solution. Finally, in Section 6 we give an example of a periodic structure which produces a nontrivial large effective convection.

\section{Setting of the Problem}

We start this section with the construction of a perforated domain depending on time. Suppose we are given a family of diffeomorphisms of $\mathbb{R}^{n}$ onto itself

$$
F_{t}: \mathbb{R}^{n} \longmapsto \mathbb{R}^{n}, \quad t \in(-\infty,+\infty),
$$

that commute with all the integer shifts along a standard basis $\left\{e_{1}, e_{2}, \ldots, e_{n}\right\}$ in $\mathbb{R}^{n}$ :

$$
F_{t}(x+k)=F_{t}(x)+k, \quad \forall x \in \mathbb{R}^{n}, \quad k \in \mathbb{Z}^{n} .
$$

Here we used the the usual notation $\mathbb{Z}^{n}$ for the integer lattice in $\mathbb{R}^{n}$. Assume moreover, that the family $F_{t}$ is smooth and 1-periodic in $t$.

Denote by $B_{0}$ the ball centered at the origin of radius $1 / 4$, and by $B$ the following union:

$$
B=\bigcup_{k \in \mathbb{Z}^{n}}\left(B_{0}+k\right) .
$$

Let us now introduce for each $t \in R$, the set

$$
G(t)=F_{t}\left(\mathbb{R}^{n} \backslash B\right) .
$$

By definition one has

$$
G(t)=G(t+1), \quad \text { for any } t \in(-\infty,+\infty) .
$$

Now, the "pulsing" perforated domain $Q_{T}^{\varepsilon}$ is defined as follows:

$$
Q_{T}^{\varepsilon}=\left\{(x, t) \in \mathbb{R}^{n+1} \mid 0 \leq t \leq T, \quad \frac{x}{\varepsilon} \in G\left(\frac{t}{\varepsilon^{2}}\right)\right\},
$$

where $\varepsilon$ is a positive parameter such that $\varepsilon \rightarrow 0$. 
Let us consider the following initial boundary problem:

$$
\left\{\begin{array}{l}
\frac{\partial u^{\varepsilon}}{\partial t}-\Delta u^{\varepsilon}=f \quad \text { in } Q_{T}^{\varepsilon} \\
\frac{\partial u^{\varepsilon}}{\partial n^{\varepsilon}}=0 \quad \text { on } \partial Q_{T}^{\varepsilon} \\
\left.u^{\varepsilon}\right|_{t=0}=g \quad \text { in } \varepsilon G(0)
\end{array}\right.
$$

Here $n^{\varepsilon}=n^{\varepsilon}(x, t)$ is the normal vector to $\partial Q_{T}^{\varepsilon}$ in the cross-section $t=$ const, i.e., the normal to the boundary $\varepsilon \partial G\left(t / \varepsilon^{2}\right)$.

In the sequel we will suppose that the functions $f$ and $g$ are such that

$$
\left\{\begin{array}{l}
f=f(x, t) \text { and } g=g(x) \text { are sufficiently smooth } \\
f \text { and } g \text { decay rapidly as }|x| \rightarrow \infty
\end{array}\right.
$$

As will be shown in Section 5 below, this assumption is essential for constructing the asymptotic expansion of $u^{\varepsilon}$ with respect to $\varepsilon$. The case of general nonsmooth data is also discussed in Section 5 ; the corresponding homogenization results are based on the approximation and a priori estimates below.

The existence and uniqueness of the solutions of problem (1.3), for each $\varepsilon>0$, is a straightforward consequence of the following simple assertion:

Proposition 1.1. Suppose that $f$ and $g$ satisfy assumption (1.4). Then, for any $\varepsilon>0$, problem (1.3) admits a unique smooth solution.

Proof. Let us do the following change of variables in (1.3):

$$
x=\varepsilon F_{t / \varepsilon^{2}}\left(\frac{y}{\varepsilon}\right)
$$

Note that the inverse function

$$
y=\varepsilon F_{t / \varepsilon^{2}}^{-1}\left(\frac{x}{\varepsilon}\right)
$$

maps $\varepsilon G\left(t / \varepsilon^{2}\right)$ onto $\varepsilon\left(\mathbb{R}^{n} \backslash B\right)$. Consequently, in the new variables $y$, equation (1.3) can be written in the form

$$
\left\{\begin{array}{l}
\frac{\partial u^{\varepsilon}}{\partial t}-a_{i j}^{\varepsilon} \frac{\partial^{2} u^{\varepsilon}}{\partial y_{i} \partial y_{j}}-b_{i}^{\varepsilon} \frac{\partial u^{\varepsilon}}{\partial y_{i}}=\widetilde{f} \quad \text { in } \varepsilon\left(\mathbb{R}^{n} \backslash B\right) \times(0, T) \\
\frac{\partial u^{\varepsilon}}{\partial n_{a}^{\varepsilon}}=0 \quad \text { on } \varepsilon \partial B \\
\left.u^{\varepsilon}\right|_{t=0}=\widetilde{g} \quad \text { in } \varepsilon\left(\mathbb{R}^{n} \backslash B\right),
\end{array}\right.
$$

where $\tilde{f}(y)=f(x(y)), \widetilde{g}(y)=g(x(y))$, and $n_{a}^{\varepsilon}=n_{a}^{\varepsilon}(y, t)$ is the conormal vector to $\varepsilon \partial B$. The coefficients $b_{i}^{\varepsilon}$ are smooth periodic functions and $a_{i j}^{\varepsilon}$ are defined by

$$
a_{i j}^{\varepsilon}(y, t)=\sum_{l=1}^{n} \frac{\partial y_{i}}{\partial x_{l}} \frac{\partial y_{j}}{\partial x_{l}}
$$

Observe that the geometry of the set where (1.5) is stated is now independent of the time variable, and therefore, uniqueness and existence result are obtained in a standard way. For details, we refer the reader for instance, to $[7]$. 
Remark 1.2. Under assumption (1.4), $u^{\varepsilon}$ is a classical solution, decaying rapidly as well as its derivatives, as $|x| \rightarrow \infty$. If $f \in L^{2}\left((0, T) \times \mathbb{R}^{n}\right)$ and $g \in L^{2}\left(\mathbb{R}^{n}\right)$, then again standard results show that (1.5) has a unique solution such that

$$
u^{\varepsilon} \in L^{2}\left(0, T ; H^{1}\left(G\left(\frac{t}{\varepsilon^{2}}\right)\right) \cap L^{\infty}\left(0, T ; L^{2}\left(G\left(\frac{t}{\varepsilon^{2}}\right)\right)\right) .\right.
$$

In both cases we cannot provide estimates of $u^{\varepsilon}$, uniform with respect to $\varepsilon$.

Remark 1.3. The geometry independent of time (as studied in [4-6] and [8]) is contained in Definition 1.2. Indeed, if $F_{t}$ does not depend on $t$, then the set $Q_{T}^{\varepsilon}$ in problem (1.3) is reduced to a usual perforated domain $Q_{T}^{\varepsilon}=G^{\varepsilon} \times(0, T)$ with $G^{\varepsilon}=\varepsilon G(0)$.

\section{Auxiliary eigenvalue problems}

In this section we consider an auxiliary eigenvalue problem whose ground state will be essential in the further analysis.

Abusing slightly of the notation, we identify the periodic set $G(\tau)$ defined in the preceding section by (1.1), with a corresponding subset of the $n$-dimensional torus $T^{n}$. Then, taking into account the periodicity of $G(\tau)$ in $\tau$, we set

$$
G=\left\{(z, \tau) \in T^{n+1}: z \in G(\tau)\right\} .
$$

Similarly, a space of 1-periodic functions in $\mathbb{R}^{n+1}\left(\right.$ or $\mathbb{R}^{n}$ ) can be identified with the corresponding functional space on the torus.

Remark 2.1. In the standard homogenization in periodically perforated domains, the set $G$ is nothing else but the usual (reference) perforated cell.

Remark 2.2. In the sequel, for a 1-periodic function $\psi=\psi(x, t)$ in $\mathbb{R}^{n+1}\left(\right.$ or $\mathbb{R}^{n}$ ), we denote by $\int_{G} \psi(z, \tau) \mathrm{d} z \mathrm{~d} \tau$ the integral of $\psi$ over $T^{n+1} \cap G$ (or over $T^{n} \cap G(\tau)$ ). This means that actually the integral is taken over one periodicity cell, i.e.,

$$
\int_{[0,1]^{n+1} \cap G} \varphi(z, \tau) \mathrm{d} z \mathrm{~d} \tau=\int_{G} \varphi(z, \tau) \mathrm{d} z \mathrm{~d} \tau .
$$

Also, given a periodic function, its integral over a subset $A$ of $T^{n+1}$, coincides with the integral over $A$ as subset of the periodicity cell $[0,1]^{n+1}$.

Consider the problem

$$
\left\{\begin{array}{l}
\frac{\partial v}{\partial \tau}-\Delta v=0 \quad \text { in } G \\
\partial v \partial n=0 \quad \text { on } \partial G
\end{array}\right.
$$

and its adjoint problem

$$
\left\{\begin{array}{l}
\frac{\partial p}{\partial \tau}+\Delta p=0 \quad \text { in } G \\
\frac{\partial p}{\partial n_{z}}+n_{\tau} p=0 \quad \text { on } \partial G,
\end{array}\right.
$$

where $n=n(z, \tau)$ is the external normal to $\partial G(\tau)$ for a given $\tau$, i.e. the normal constructed in $z$-variables for a fixed $\tau$, while $n_{z}$ and $n_{\tau}$ are respectively, the $z$ - and $\tau$-coordinates of the total $(n+1)$-dimensional external normal to $\partial G$. in $\tau$.

Problems (2.1) and (2.2) make sense since, by construction, we are looking for solutions that are 1-periodic 
Remark 2.3. Observe that the only solution of $(2.1)$ is a constant. Indeed, in the coordinates $(y, \tau)=$ $\left(F_{\tau}^{-1}(z), \tau\right)$, equation $(2.1)$ has the form

$$
\left\{\begin{array}{l}
\frac{\partial v}{\partial \tau}-a_{i j} \frac{\partial^{2} v}{\partial y_{i} \partial y_{j}}-b_{i} \frac{\partial v}{\partial y_{i}}=0 \quad \text { in }\left(T^{n} \backslash B\right) \times T^{1} \\
\frac{\partial v}{\partial n_{a}}=0 \quad \text { on } \partial B \times T^{1}
\end{array}\right.
$$

where

$$
a_{i j}(y, \tau)=\sum_{l=1}^{n} \frac{\partial y_{i}}{\partial x_{l}} \frac{\partial y_{j}}{\partial x_{l}}, \quad b_{i}(y, \tau)=\sum_{l=1}^{n} \frac{\partial^{2} y_{i}}{\partial x_{l}^{2}}-\frac{\partial y_{i}}{\partial \tau}
$$

and $n_{a}$ is the conormal vector. Now the required statement follows from the standard maximum principle.

The study of problem (2.2) requires more delicate arguments because of the presence of zero-order terms in the boundary condition. This does not allow us to use the same reasoning as that in Remark 2.3. As a matter of fact, we have the following result:

Proposition 2.4. Problem (2.2) has a unique (up to a multiplicative constant) solution. This solution is positive and smooth.

Proof. Consider the auxiliary initial-boundary problem

$$
\left\{\begin{array}{l}
\frac{\partial q}{\partial \tau}+\Delta q=0 \quad \text { in } G_{\text {neg }}=\{(z, \tau): \tau \in(-\infty, 0), z \in G(\tau)\} \\
\frac{\partial q}{\partial n_{z}}+n_{\tau} q=0 \quad \text { on } \partial G_{\text {neg }} \\
\left.q\right|_{t=0}=\varphi \quad \text { in } G(0)
\end{array}\right.
$$

where $\varphi=\varphi(z)$ is a smooth function. This problem is stated in a half-infinite in time set with a "perforated" torus in a cross-section.

By virtue of the same change of variables $(y, \tau)=\left(F_{\tau}^{-1}(z), \tau\right)$, we reduce problem (2.4) to an initial-boundary problem for a uniformly parabolic operator in the direct product $\left(T^{n} \backslash B\right) \times(-\infty, 0)$ with Fourier boundary condition on $\partial B \times(-\infty, 0)$. This transformed problem is standard, and the existence and uniqueness of its solution is well-known. Thus problem (2.4) is also well-posed.

Then we can define a family of operators

$$
S_{\tau}: \varphi(\cdot) \longmapsto q(\cdot, \tau), \quad \text { for }-\infty<\tau \leq 0,
$$

that map the initial condition into the solution of (2.4) at the time $\tau$.

The analysis of problem (2.2) is essentially based on the properties of the operator $S_{-1}$. Let us study this operator in detail. Note first that, thanks to the periodicity of $G(\tau)$ in $\tau, S_{-1}$ maps the spaces $L^{2}(G(0))$ and $C(G(0))$ in themselves. Moreover, in view of the smoothness properties of the solutions of $(2.2), S_{-1}$ is compact in both spaces $L^{2}(G(0))$ and $C(G(0))$. Finally, by the maximum principle, the function $S_{-1} \varphi(\cdot)$ is positive if $\varphi$ is positive.

Observe that by [12], the principal eigenvalue $\lambda_{0}$ of $S_{-1}$ is real, positive and simple, and the corresponding eigenfunction $p_{0}=p_{0}(z)$ is real and positive. Moreover, the rest of the spectrum of $S_{-1}$ belongs to a disk $\left\{|\lambda|<\lambda_{0}-\delta\right\}$ for some $\delta>0$. 
We would like to show that $\lambda_{0}=1$. To this end, let us analyze the behaviour of the integral of $q$ over a cross-section of $G_{\text {neg. }}$. Integrating by parts gives successively,

$$
\begin{aligned}
\int_{G\left(\tau_{2}\right)} q\left(z, \tau_{2}\right) \mathrm{d} z-\int_{G\left(\tau_{1}\right)} q\left(z, \tau_{1}\right) \mathrm{d} z & =\int_{\tau_{1}}^{\tau_{2}} \int_{G(\tau)} \frac{\partial q(z, \tau)}{\partial \tau} \mathrm{d} z \mathrm{~d} \tau-\int_{\tau_{1}}^{\tau_{2}} \int_{\partial G(\tau)} q(z, \tau) n_{\tau} \mathrm{d} s_{z, \tau} \\
& =-\int_{\tau_{1}}^{\tau_{2}} \int_{G(\tau)} \Delta q \mathrm{~d} z \mathrm{~d} \tau-\int_{\tau_{1}}^{\tau_{2}} \int_{\partial G(\tau)} q(z, \tau) n_{\tau} \mathrm{d} s_{z, \tau} \\
& =-\int_{\tau_{1}}^{\tau_{2}} \int_{\partial G(\tau)}\left(\frac{\partial q}{\partial n_{z}}+q(z, \tau) n_{\tau}\right) \mathrm{d} s_{z, \tau}=0 .
\end{aligned}
$$

Consequently, the operator $S_{-1}$ preserves the integral over $G(0)$. Since the first eigenfunction is positive, this implies that $\lambda_{0}=1$. This means in turn, that the solution $p=p(z, \tau)$ of problem (2.4) with initial data $p_{0}=p_{0}(z)$, is a 1-periodic function of $\tau$. Thus, this $p$ is nothing else but a solution of (2.2).

The uniqueness of $p$ follows immediately from the properties of the operator $S_{-1}$, while its smoothness is a consequence of standard parabolic estimates. This ends the proof of Proposition 2.4.

In order to fix the choice of the function $p$, we will impose the following normalization condition

$$
\int_{G} p(z, \tau) \mathrm{d} z \mathrm{~d} \tau=1 .
$$

Remark 2.5. From the above considerations, it follows that the operator $\left(I d-S_{-1}\right)$ has a one-dimensional kernel, generated by the function $p_{0}$.

Now, let us consider the periodic auxiliary problem with the nonhomogeneous data $\Psi=\Psi(z, \tau)$ and $\Phi=$ $\Phi(z, \tau)$

$$
\left\{\begin{array}{l}
\frac{\partial v}{\partial \tau}-\Delta v=\Psi \quad \text { in } G \\
\frac{\partial v}{\partial n}=\Phi \quad \text { on } \partial G
\end{array}\right.
$$

The compatibility condition for this problem is given by the following statement:

Proposition 2.6. Problem (2.7) is solvable if and only if the following equality holds:

$$
\int_{G} \Psi(z, \tau) p(z, \tau) \mathrm{d} z \mathrm{~d} \tau+\int_{\partial G} \Phi(z, \tau) p(z, \tau) \mathrm{d} s_{z} \mathrm{~d} \tau=0,
$$

where $p$ is the solution of problem (2.2).

Proof. Let us first show that (2.8) is necessary. Suppose that $v$ satisfies (2.7) and let $p$ be the solution of (2.2). By periodicity, we have

$$
\int_{G(1)} v(z, 1) p(z, 1) \mathrm{d} z-\int_{G(0)} v(z, 0) p(z, 0) \mathrm{d} z=0 .
$$


By integrating by parts and using equations (2.2) and (2.7), we get successively,

$$
\begin{aligned}
0= & \int_{G} \frac{\partial[v(z, \tau) p(z, \tau)]}{\partial \tau} \mathrm{d} z \mathrm{~d} \tau-\int_{\partial G} v(z, \tau) p(z, \tau) n_{\tau} \mathrm{d} s_{z} \mathrm{~d} \tau \\
= & \int_{G} p(z, \tau) \Delta v(z, \tau) \mathrm{d} z \mathrm{~d} \tau-\int_{G} v(z, \tau) \Delta p(z, \tau) \mathrm{d} z \mathrm{~d} \tau \\
& +\int_{G} \Psi(z, \tau) p(z, \tau) \mathrm{d} z \mathrm{~d} \tau-\int_{\partial G} v(z, \tau) p(z, \tau) n_{\tau} \mathrm{d} s_{z} \mathrm{~d} \tau,
\end{aligned}
$$

whence, by Green's formula

$$
\begin{aligned}
0= & -\int_{\partial G} v(z, \tau)\left[\frac{\partial p(z, \tau)}{\partial n_{z}}+p(z, \tau) n_{\tau}\right] \mathrm{d} s_{z, \tau} \\
& +\int_{\partial G} p(z, \tau) \Phi(z, \tau) \mathrm{d} s_{z} \mathrm{~d} \tau+\int_{G} \Psi(z, \tau) p(z, \tau) \mathrm{d} z \mathrm{~d} \tau \\
= & \int_{\partial G} p(z, \tau) \Phi(z, \tau) \mathrm{d} s_{z} \mathrm{~d} \tau+\int_{G} \Psi(z, \tau) p(z, \tau) \mathrm{d} z \mathrm{~d} \tau .
\end{aligned}
$$

Now, suppose that $\Psi$ and $\Phi$ satisfy (2.8), and let $\widehat{v}$ be the solution of the following initial boundary problem:

$$
\left\{\begin{array}{l}
\frac{\partial \widehat{v}}{\partial \tau}-\Delta \widehat{v}=\Psi \quad \text { in }\{(z, \tau) \mid z \in G(\tau), \quad \tau \in(0,+\infty)\} \\
\frac{\partial \widehat{v}}{\partial n}=\Phi \quad \text { on } \partial G \\
\left.\widehat{v}\right|_{t=0}=0 \quad \text { in } G(0)
\end{array}\right.
$$

Integrating by parts like in (2.9) and (2.10), we get

$$
\int_{G(0)} \widehat{v}(z, 1) p_{0}(z) \mathrm{d} z=\int_{G(1)} \widehat{v}(z, 1) p(z, 1) \mathrm{d} z-\int_{G(0)} \widehat{v}(z, 0) p(z, 0) \mathrm{d} z=0
$$

where we have used the fact that

$$
p_{0}(z)=p(z, 0)=p(z, 1) .
$$

From (2.12) it follows that $\widehat{v}(z, 1)$ is orthogonal in $L^{2}(G(0))$, to the kernel $p_{0}$ of the operator $\left(I d-S_{-1}\right)$ (see Rem. 2.5 above). In view of the compactness of $S_{-1}$, this implies the solvability of the equation

$$
\left(I d-S_{-1}^{\star}\right) \widetilde{v}(z)=\widehat{v}(z, 1)
$$

Now, let us prove that

where $\bar{v}=\bar{v}(z, \tau)$ solves the problem

$$
S_{-1}^{\star} \widetilde{v}(z)=\bar{v}(z, 1)
$$

$$
\left\{\begin{array}{l}
\frac{\partial \bar{v}}{\partial \tau}-\Delta \bar{v}=0 \quad \text { in }\{(z, \tau) \mid \tau \in(0,+\infty), z \in G(\tau)\} \\
\frac{\partial \bar{v}}{\partial n}=0 \quad \text { on } \partial G \\
\left.\bar{v}\right|_{t=0}=\widetilde{v} \quad \text { in } G(0)
\end{array}\right.
$$


This means that $S_{-1}^{\star}$ can be expressed in terms of a solution of the problem adjoint to (2.4), with time going in the positive direction.

To prove (2.14), it is sufficient to show that

$$
\int_{G(1)} \varphi(z) \bar{v}(z, 1) \mathrm{d} z=\int_{G(0)} S_{-1} \varphi(z) \widetilde{v}(z) \mathrm{d} z,
$$

for any smooth function $\varphi$. To do so, let $q=q(z, \tau)$ be the solution of (2.4) with data $\varphi$. By the definition (2.5) of the operator $S_{-1}$,

$$
S_{-1} \varphi(z)=q(z,-1) \text {. }
$$

Then, due to the initial conditions in (2.4) and (2.15), we have

$$
\begin{aligned}
I & =\int_{G(1)} \varphi(z) \bar{v}(z, 1) \mathrm{d} \tau-\int_{G(0)} S_{-1} \varphi(z) \widetilde{v}(z) \mathrm{d} \tau \\
& =\int_{G(1)} \varphi(z) \bar{v}(z, 1) \mathrm{d} \tau-\int_{G(0)} q(z,-1) \bar{v}(z, 0) \mathrm{d} \tau .
\end{aligned}
$$

Integrating by parts and considering the equations from (2.15) and (2.4), we derive that

$$
\begin{aligned}
I= & \int_{G} \frac{\partial[q(z, \tau+1) \bar{v}(z, \tau)]}{\partial \tau} \mathrm{d} z \mathrm{~d} \tau-\int_{\partial G} q(z, \tau+1) \bar{v}(z, \tau) n_{\tau} \mathrm{d} s_{z} \mathrm{~d} \tau \\
= & \int_{G} q(z, \tau+1) \Delta \bar{v}(z, \tau) \mathrm{d} z \mathrm{~d} \tau-\int_{G} \bar{v}(z, \tau) \Delta q(z, \tau+1) \mathrm{d} z \mathrm{~d} \tau \\
& -\int_{\partial G} q(z, \tau+1) \bar{v}(z, \tau) n_{\tau} \mathrm{d} s_{z} \mathrm{~d} \tau .
\end{aligned}
$$

Finally, Green's formula and the boundary conditions in (2.4) and (2.15) yield

$$
I=\int_{\partial G} q(z, \tau+1) \frac{\partial \bar{v}(z, \tau)}{\partial n_{z}} \mathrm{~d} s_{z, \tau}+\int_{\partial G} \bar{v}(z, \tau)\left[\frac{\partial q(z, \tau+1)}{\partial n_{z}}+q(z, \tau+1) n_{\tau}\right] \mathrm{d} s_{z, \tau}=0 .
$$

Hence (2.16) and consequently (2.14), are proved.

To conclude the proof of Proposition 2.6, let us set

$$
v=\widehat{v}+\bar{v} .
$$

This $v$ is the required solution of (2.7). Recalling that $\widehat{v}$ and $\bar{v}$ are the solutions of problems (2.11) and (2.15) respectively, it is obvious that $v$ satisfies the equation and the boundary condition in (2.7).

It remains to show that $v$ is 1-periodic in $\tau$. To do so, observe that by using successively (2.13) and (2.14), we have

$$
v(z, 1)=\widehat{v}(z, 1)+\bar{v}(z, 1)=\widetilde{v}(z)-S_{-1}^{\star} \widetilde{v}(z)+\widetilde{v}(z, 1)=\widetilde{v}(z) .
$$

But from the initial conditions in problems (2.11) and (2.14), one has

$$
\widetilde{v}(z)=\widehat{v}(z, 0)+\bar{v}(z, 0)=v(z, 0),
$$

and this ends the proof of Proposition 2.6. 


\section{Energy estimates}

This section is devoted to qualified energy estimates for problem (1.3) and for the following problem with more general data than (1.3):

To simplify the notation we set

$$
\left\{\begin{array}{l}
\frac{\partial v^{\varepsilon}}{\partial t}-\Delta v^{\varepsilon}=f \quad \text { in } Q_{T}^{\varepsilon} \\
\left.\frac{\partial v^{\varepsilon}}{\partial n^{\varepsilon}}\right|_{\partial Q_{T}^{\varepsilon}}=\psi \quad \text { in } \partial Q_{T}^{\varepsilon} \\
\left.v^{\varepsilon}\right|_{t=0}=g \quad \text { in } \varepsilon G(0) .
\end{array}\right.
$$

$$
G^{\varepsilon}(t)=\varepsilon G\left(\frac{t}{\varepsilon^{2}}\right)
$$

(see definitions (1.1) and (1.2) of domains $G(t)$ and $Q_{T}^{\varepsilon}$, respectively).

Proposition 3.1. Let $f \in L^{2}\left(\mathbb{R}^{n} \times(0, T)\right), g \in L^{2}\left(\mathbb{R}^{n}\right)$ and $\psi \in L^{2}\left(\partial Q_{T}^{\varepsilon}\right)$. Then the solution of problem (1.3) satisfies the following estimate:

$$
\int_{Q_{T}^{\varepsilon}}\left|\nabla u^{\varepsilon}(x, t)\right|^{2} \mathrm{~d} x \mathrm{~d} t+\int_{G^{\varepsilon}(T)}\left[u^{\varepsilon}(x, T)\right]^{2} \mathrm{~d} x \leq C\left(\|f\|_{L^{2}\left(\mathbb{R}^{n} \times(0, T)\right)}^{2}+\|g\|_{L^{2}\left(\mathbb{R}^{n}\right)}^{2}\right)
$$

while the solution of problem (3.1) satisfies the estimate

$$
\int_{Q_{T}^{\varepsilon}}\left|\nabla v^{\varepsilon}(x, t)\right|^{2} \mathrm{~d} x \mathrm{~d} t+\int_{G_{t}^{\varepsilon}}\left[v^{\varepsilon}(x, T)\right]^{2} \mathrm{~d} x \leq C\left(\|f\|_{L^{2}\left(\mathbb{R}^{n} \times(0, T)\right)}^{2}+\|g\|_{L^{2}\left(\mathbb{R}^{n}\right)}^{2}+\varepsilon^{-1}\|\psi\|_{L^{2}\left(\partial Q_{T}^{\varepsilon}\right)}^{2}\right) .
$$

If moreover, $\psi$ is in $L^{2}\left(0, T ; H^{1}\left(G^{\varepsilon}(\cdot)\right)\right)$, then the following estimate holds:

$$
\int_{Q_{T}^{\varepsilon}}\left|\nabla v^{\varepsilon}(x, t)\right|^{2} \mathrm{~d} x \mathrm{~d} t+\int_{G_{t}^{\varepsilon}}\left[v^{\varepsilon}(x, T)\right]^{2} \mathrm{~d} x \leq C\left(\|f\|_{L^{2}\left(\mathbb{R}^{n} \times(0, T)\right)}^{2}+\|g\|_{L^{2}\left(\mathbb{R}^{n}\right)}^{2}+\varepsilon^{-2}\|\psi\|_{L^{2}\left(0, T ; H^{1}\left(G^{\varepsilon}(\cdot)\right)\right)}^{2}\right) .
$$

In all these estimates, the constant $C$ is independent of $\varepsilon$.

Proof. Let $p=p(x, t)$ be the solution of problem (2.2). Multiplying the equation in (3.1) by $v^{\varepsilon}(x, t) p\left(x / \varepsilon, t / \varepsilon^{2}\right)$ and integrating by parts, we obtain after simple transformations

$$
\begin{aligned}
\int_{Q_{T}^{\varepsilon}} p\left(\frac{x}{\varepsilon}, \frac{t}{\varepsilon^{2}}\right)\left|\nabla v^{\varepsilon}(x, t)\right|^{2} \mathrm{~d} x \mathrm{~d} t & +\int_{G^{\varepsilon}(T)} p\left(\frac{x}{\varepsilon}, \frac{T}{\varepsilon^{2}}\right)\left[v^{\varepsilon}(T, x)\right]^{2} \mathrm{~d} x=\int_{Q_{T}^{\varepsilon}} p\left(\frac{x}{\varepsilon}, \frac{t}{\varepsilon^{2}}\right) v^{\varepsilon}(x, t) f(x, t) \mathrm{d} x \mathrm{~d} t \\
& +\int_{G^{\varepsilon}(0)} p\left(\frac{x}{\varepsilon}, 0\right) g^{2}(x) \mathrm{d} x+\int_{\partial Q_{T}^{\varepsilon}} p\left(\frac{x}{\varepsilon}, \frac{t}{\varepsilon^{2}}\right) \psi(x, t) v^{\varepsilon}(x, t) \mathrm{d} s_{x} \mathrm{~d} t .
\end{aligned}
$$

For problem (1.3), relation (3.5) implies immediately estimate (3.2) with a constant $C$ independent of $\varepsilon$. In the case of problem (3.1), let us introduce the rescaled coordinates

$$
\tau=\frac{t}{\varepsilon^{2}}, \quad z=\frac{x}{\varepsilon}
$$


Observe that in these new coordinates, for any sufficiently smooth function $U=U(x, t)$, we have

$$
\int_{\partial G(\tau)}\left[U\left(\varepsilon z, \varepsilon^{2} \tau\right)\right]^{2} \mathrm{~d} s_{z} \leq c\left(\int_{G(\tau)}\left[U\left(\varepsilon z, \varepsilon^{2} \tau\right)\right]^{2} \mathrm{~d} z+\int_{G(\tau)}\left|\nabla_{z} U\left(\varepsilon z, \varepsilon^{2} \tau\right)\right|^{2} \mathrm{~d} z\right),
$$

since the shape of the cross-section $\left\{(z, \tau): \tau=\right.$ const, $\left.\left(\varepsilon z, \varepsilon^{2} \tau\right) \in Q_{T}^{\varepsilon}\right\}$ does not depend on the parameter $\varepsilon$. Integrating this estimate in $\tau$ and going back to the initial variables, we obtain the following bound:

$$
\int_{\partial Q_{T}^{\varepsilon}} U^{2}(x, t) \mathrm{d} s_{x, t} \leq c\left(\varepsilon^{-1} \int_{Q_{T}^{\varepsilon}} U^{2}(x, t) \mathrm{d} \tau+\varepsilon \int_{Q_{T}^{\varepsilon}}\left|\nabla_{x} U(x, t)\right|^{2} \mathrm{~d} \tau\right) .
$$

Let $v^{\varepsilon}$ be the solution of problem (3.1). Then by the Schwarz inequality and using (3.6), one gets

$$
\begin{aligned}
\left|\int_{\partial Q_{T}^{\varepsilon}} v^{\varepsilon}(x, t) \psi(x, t) \mathrm{d} s_{x, t}\right| & \leq\left(\int_{\partial Q_{T}^{\varepsilon}}\left[v^{\varepsilon}(x, t)\right]^{2} \mathrm{~d} s_{x, t}\right)^{1 / 2}\left(\int_{\partial Q_{T}^{\varepsilon}} \psi^{2}(x, t) \mathrm{d} s_{x, t}\right)^{1 / 2} \\
& \leq \varepsilon^{-1 / 2}\left\|v^{\varepsilon}\right\|_{L^{2}\left(0, T ; H^{1}\left(G^{\varepsilon}(\cdot)\right)\right)}\|\psi\|_{L^{2}\left(\partial Q_{T}^{\varepsilon}\right)}
\end{aligned}
$$

which used in (3.5) gives easily estimate (3.3) with a constant independent of $\varepsilon$.

Suppose now that the boundary data $\psi$ in (3.1), is the restriction to $\partial Q_{T}^{\varepsilon}$ of a function from $L^{2}\left(0, T ; H^{1}\left(G^{\varepsilon}(\cdot)\right)\right)$. For such a case, inequality (3.7) can be modified as follows:

$$
\left|\int_{\partial Q_{T}^{\varepsilon}} v^{\varepsilon}(x, t) \psi(x, t) \mathrm{d} s_{x, t}\right| \leq \varepsilon^{-1}\left\|v^{\varepsilon}\right\|_{L^{2}\left(0, T ; H^{1}\left(G^{\varepsilon}(\cdot)\right)\right)}\|\psi\|_{L^{2}\left(0, T ; H^{1}\left(G^{\varepsilon}(\cdot)\right)\right)},
$$

which, together with (3.5) and (3.7), leads to estimate (3.4). This ends the proof of Proposition 3.1.

\section{Formal ASYMPTOTIC EXPANSIONS}

The aim of the section is to construct a formal asymptotic expansion for the solution of problem (1.3). To do so, we make use of the ideas of the multiple scale asymptotic decomposition method (see, for example [1,2]). However, the presence of moving boundaries suggests that for small $\varepsilon$, the problem might involve large convection terms. In order to take them into account, we construct the principal terms of the expansion in the following form:

$$
\begin{aligned}
u^{\varepsilon}(x, t) \sim u_{0}\left(x-\frac{\alpha}{\varepsilon} t, t\right)+ & \varepsilon\left\{\nabla_{x} u_{0}\left(x-\frac{\alpha}{\varepsilon} t, t\right) \chi\left(\frac{x}{\varepsilon}, \frac{t}{\varepsilon^{2}}\right)+u_{1}\left(x-\frac{\alpha}{\varepsilon} t, t\right)\right\} \\
& +\varepsilon^{2}\left\{\nabla_{x} \nabla_{x} u_{0}\left(x-\frac{\alpha}{\varepsilon} t, t\right) \xi\left(\frac{x}{\varepsilon}, \frac{t}{\varepsilon^{2}}\right)+\nabla_{x} u_{0}\left(x-\frac{\alpha}{\varepsilon} t, t\right) \chi\left(\frac{x}{\varepsilon}, \frac{t}{\varepsilon^{2}}\right)\right\}+\cdots
\end{aligned}
$$

where $\chi$ and $\xi$ are respectively, a periodic vector-function and a periodic matrix-function, and $\alpha$ is a constant vector, $\alpha=\left(\alpha_{1}, \ldots, \alpha_{n}\right)$. 
Note that $\left((x-(\alpha / \varepsilon), t)\right.$ plays the role of slow variables, while $z=x / \varepsilon$ and $\tau=t / \varepsilon^{2}$, are the fast ones. Recall that the basic idea of the multiple scale asymptotic decomposition method, is to suppose that the slow and the fast variables are independent.

In the sequel, we will use systematically the following derivation formulae:

$$
\left\{\begin{array}{l}
\frac{\partial}{\partial x} g\left(x, \frac{x}{\varepsilon}\right)=\left.\left(\frac{\partial g(x, z)}{\partial x}+\varepsilon^{-1} \frac{\partial g(x, z)}{\partial z}\right)\right|_{z=x / \varepsilon} \\
\frac{\partial}{\partial t} g\left(t, \frac{t}{\varepsilon^{2}}\right)=\left.\left(\frac{\partial g(t, \tau)}{\partial t}+\varepsilon^{-2} \frac{\partial g(t, \tau)}{\partial \tau}\right)\right|_{\tau=t / \varepsilon^{2}}
\end{array}\right.
$$

Substituting (4.1) in (1.3), applying (4.2) and equating the power-like terms of $\varepsilon$, we obtain the system

$$
\left\{\begin{array}{l}
{\left[\frac{\partial \chi(z, \tau)}{\partial \tau}-\Delta_{z} \chi(z, \tau)\right] \nabla_{x} u_{0}\left(x-\frac{\alpha}{\varepsilon} t, t\right)=-\alpha \nabla_{x} u_{0}\left(x-\frac{\alpha}{\varepsilon} t, t\right)} \\
\frac{\partial \chi(z, \tau)}{\partial n} \nabla_{x} u_{0}\left(x-\frac{\alpha}{\varepsilon} t, t\right)+n(z, \tau) \nabla_{x} u_{0}\left(x-\frac{\alpha}{\varepsilon} t, t\right)=0 .
\end{array}\right.
$$

For $\ell=1, \ldots, n$, let $\chi_{\ell}=\chi_{\ell}(z, \tau)$ be a solution of the problem

$$
\left\{\begin{array}{l}
\frac{\partial \chi_{\ell}}{\partial \tau}-\Delta \chi_{\ell}=-\alpha_{\ell} \quad \text { in } G \\
\frac{\partial \chi_{\ell}}{\partial n}=-n_{\ell} \quad \text { on } \partial G
\end{array}\right.
$$

By Proposition 2.6, the compatibility condition in the last equation reads

$$
\alpha_{\ell}=-\int_{\partial G} p(z, \tau) n_{\ell}(z, \tau) \mathrm{d} s_{z} \mathrm{~d} \tau
$$

At the next step of the identification, we get

$$
\left\{\begin{array}{c}
{\left[\frac{\partial \xi(z, \tau)}{\partial \tau}-\Delta_{z} \xi(z, \tau)\right] \nabla_{x} \nabla_{x} u_{0}\left(x-\frac{\alpha}{\varepsilon} t, t\right)+\left.\left(\frac{\partial}{\partial s} u_{0}\left(x-\frac{\alpha}{\varepsilon} t, s\right)\right)\right|_{s=t}} \\
-\Delta_{x} u_{0}\left(x-\frac{\alpha}{\varepsilon} t, t\right)+2 \nabla_{z} \chi(z, \tau) \nabla_{x} \nabla_{x} u_{0}\left(x-\frac{\alpha}{\varepsilon} t, t\right) \\
-\alpha \chi(z, \tau) \nabla_{x} \nabla_{x} u_{0}\left(x-\frac{\alpha}{\varepsilon} t, t\right) \\
+\left[\frac{\partial \chi(z, \tau)}{\partial \tau}-\Delta_{z} \chi(z, \tau)-\alpha\right] \nabla_{x} u_{1}\left(x-\frac{\alpha}{\varepsilon} t, t\right)=f(x, t) \\
\frac{\partial \xi(z, \tau)}{\partial n} \nabla_{x} \nabla_{x} u_{0}\left(x-\frac{\alpha}{\varepsilon} t, t\right)+n(z, \tau) \nabla_{x} \nabla_{x} u_{0}\left(x-\frac{\alpha}{\varepsilon} t, t\right) \chi(z, \tau) \\
+\frac{\partial \chi(z, \tau)}{\partial n} \nabla_{x} u_{1}\left(x-\frac{\alpha}{\varepsilon} t, t\right)+n(z, \tau) \nabla_{x} u_{1}\left(x-\frac{\alpha}{\varepsilon} t, t\right)=0 .
\end{array}\right.
$$

The compatibility condition for (4.6) is (see again Prop. 2.6)

$$
\left.\left(\frac{\partial}{\partial s} u_{0}\left(x-\frac{\alpha}{\varepsilon} t, s\right)\right)\right|_{s=t}-a_{i j} \frac{\partial^{2}}{\partial x_{i} \partial x_{j}}\left(u_{0}\left(x-\frac{\alpha}{\varepsilon} t, t\right)\right)=f(x, t)
$$


where the constants $a_{i j}$, the "homogenized coefficients", are defined by

$$
\begin{aligned}
a_{i j}= & \delta_{i j}+\alpha_{i} \int_{G} \chi_{i}(z, \tau) p(z, \tau) \mathrm{d} z \mathrm{~d} \tau+2 \int_{G} \frac{\partial \chi_{j}(z, \tau)}{\partial z_{i}} p(z, \tau) \mathrm{d} z \mathrm{~d} \tau \\
& +\int_{\partial G} \chi_{j}(z, \tau) p(z, \tau) n_{i}(z, \tau) \mathrm{d} s_{z} \mathrm{~d} \tau .
\end{aligned}
$$

Letting

$$
U_{0}^{\varepsilon}(x, t)=u_{0}\left(x-\frac{\alpha}{\varepsilon} t, t\right),
$$

equation (4.7) can be rewritten in the form

$$
\frac{\partial U_{0}^{\varepsilon}}{\partial t}-a_{i j} \frac{\partial^{2} U_{0}^{\varepsilon}}{\partial x_{i} \partial x_{j}}+\frac{\alpha_{i}}{\varepsilon} \frac{\partial U_{0}^{\varepsilon}}{\partial x_{i}}=f \quad \text { in } \mathbb{R}^{n} \times(0, T) .
$$

In the new coordinates

$$
y=x-\frac{\alpha}{\varepsilon} t,
$$

equation (4.7) reads

$$
\frac{\partial u_{0}(y, t)}{\partial t}-a_{i j} \frac{\partial^{2} u_{0}(y, t)}{\partial y_{i} \partial y_{j}}=f\left(y+\frac{\alpha}{\varepsilon} t, t\right) \quad \text { in } \mathbb{R}^{n} \times(0, T)
$$

Considering (4.4) and (2.2), and integrating by parts in (4.8), one can rewrite $a_{i j}$ as follows:

$$
a_{i j}=\int_{G}\left(I d+\nabla_{z} \chi(z, \tau)\right) p(z, \tau)\left(I d+\nabla_{z} \chi(z, \tau)\right)^{\star} \mathrm{d} z \mathrm{~d} \tau
$$

Since the vector-function $\chi(z, \tau)$ is periodic, the matrix $a_{i j}$ defined by (4.8), is positive definite (for the proof see for instance $[2,5]$ or $[8]$ ). Therefore, equation (4.12), together with the initial condition

$$
\left.u_{0}(x, t)\right|_{t=0}=g(x)
$$

is a well-posed Cauchy problem for determining $u_{0}$.

Making one more step in the identification in problem (1.3), one can equate to zero the terms of order $\varepsilon$ in the equation and the terms of order $\varepsilon^{2}$ in the boundary condition. In this way we get an additional term in the expansion, namely the function $u_{1}$.

Let us summarize the above results in the following statement:

Theorem 4.1. Suppose that $f$ and $g$ satisfy assumption (1.4). Then the solution $u^{\varepsilon}$ of problem (1.3), can be written in the form (4.1), i.e.,

$$
u^{\varepsilon}(x, t) \sim u_{0}\left(x-\frac{\alpha}{\varepsilon} t, t\right)+\varepsilon\left\{\chi_{\ell}\left(\frac{x}{\varepsilon}, \frac{t}{\varepsilon^{2}}\right) \frac{\partial u_{0}}{\partial y_{\ell}}\left(x-\frac{\alpha}{\varepsilon} t, t\right)+u_{1}\left(x-\frac{\alpha}{\varepsilon} t, t\right)\right\}+\cdots,
$$

where $\chi_{\ell}$, for $\ell=1, \ldots, n$, satisfies problem (4.4) with $\alpha_{\ell}$ given by (4.5). 
If $\alpha \neq 0$, the function $u_{0}=u_{0}(y, t)$ is the unique solution of the limit problem

$$
\left\{\begin{array}{l}
\frac{\partial u_{0}}{\partial t}-a_{i j} \frac{\partial^{2} u_{0}}{\partial y_{i} \partial y_{j}}=0 \quad \text { in } \mathbb{R}^{n} \times(0, T) \\
\left.u_{0}\right|_{t=0}=g \text { in } \mathbb{R}^{n},
\end{array}\right.
$$

where the coefficients $a_{i j}$ are defined by (4.13).

If $\alpha=0$, the function $u_{0}=u_{0}(y, t)$ is the unique solution of the limit problem

$$
\left\{\begin{array}{l}
\frac{\partial u_{0}}{\partial t}-a_{i j} \frac{\partial^{2} u_{0}}{\partial y_{i} \partial y_{j}}=f \text { in } \mathbb{R}^{n} \times(0, T) \\
\left.u_{0}\right|_{t=0}=g \text { in } \mathbb{R}^{n} .
\end{array}\right.
$$

Corollary 4.2. Suppose that $u^{\varepsilon}$ is the solution of the problem

$$
\left\{\begin{array}{l}
\frac{\partial u^{\varepsilon}}{\partial t}-\Delta u^{\varepsilon}=f^{\varepsilon} \quad \text { in } Q_{T}^{\varepsilon} \\
\frac{\partial u^{\varepsilon}}{\partial n^{\varepsilon}}=0 \quad \text { on } \partial Q_{T}^{\varepsilon} \\
\left.u^{\varepsilon}\right|_{t=0}=g \quad \text { in } \varepsilon G(0),
\end{array}\right.
$$

where $f^{\varepsilon}$ is defined by

$$
f^{\varepsilon}(x, t)=f\left(x-\frac{\alpha}{\varepsilon}, t\right) \quad \text { in } \mathbb{R}^{n} \times(0, T)
$$

and suppose that $f$ and $g$ satisfy assumption (1.4). Then $u^{\varepsilon}$ can be written in the form (4.14) where $u_{0}$ satisfies the limit problem

$$
\left\{\begin{array}{l}
\frac{\partial u_{0}}{\partial t}-a_{i j} \frac{\partial^{2} u_{0}}{\partial y_{i} \partial y_{j}}=f \quad \text { in } \mathbb{R}^{n} \times(0, T) \\
\left.u^{\varepsilon}\right|_{t=0}=g \text { in } \mathbb{R}^{n} .
\end{array}\right.
$$

Proof. Due to assumption (1.4), the right-hand side in (4.12) vanishes as $\varepsilon \rightarrow 0$, whence (4.15).

Under hypothesis (4.16), the limit equation for $u_{0}$ preserves a non-zero function on the right-hand side. Indeed, in this case equation (4.10) reads

$$
\frac{\partial U_{0}^{\varepsilon}}{\partial t}(x, t)-a_{i j} \frac{\partial^{2} U_{0}^{\varepsilon}}{\partial x_{i} \partial x_{j}}(x, t)+\frac{\alpha_{i}}{\varepsilon} \frac{\partial U_{0}^{\varepsilon}}{\partial x_{i}}(x, t)=f^{\varepsilon}(x, t)=f\left(x-\frac{\alpha}{\varepsilon}, t\right),
$$

which, by the change of variables (4.11) and taking into account (4.9), leads immediately to system (4.17).

Remark 4.3. Notice that in the case of holes with geometry independent of time, formula (4.13) gives the classical "homogenized" coefficients (see for instance [2] and [7] and for further details [14]). Indeed, in this case, the function $p$ is equal to a constant $C$ and by the normalization condition (2.6), this constant must be $C=1 /|G|$, where $|G|$ is the volume of $G$.

Remark 4.4. In the presence of proper symmetries, the convection coefficient $\alpha$ in the limit equation is equal to 0 . We provide here two such cases:

- the set $G_{t}$, for each $t$, is symmetric with respect to some point $x_{0}$ that does not depend on $t$. It is the case of the "pulsing" structure from Figure 1;

- in the two-dimensional case, there are $t_{0} \neq 0$ and $\theta \in(0,2 \pi)$, such that for each $t$, the set $G_{\left(t+t_{0}\right)}$ coincides with the rotation of $G_{t}$ of angle $\theta$. It is the case of the "snakes-like" structure from Figure 2 . In Section 6 below, we will give an example of a geometry without such symmetries, for which $\alpha \neq 0$. 


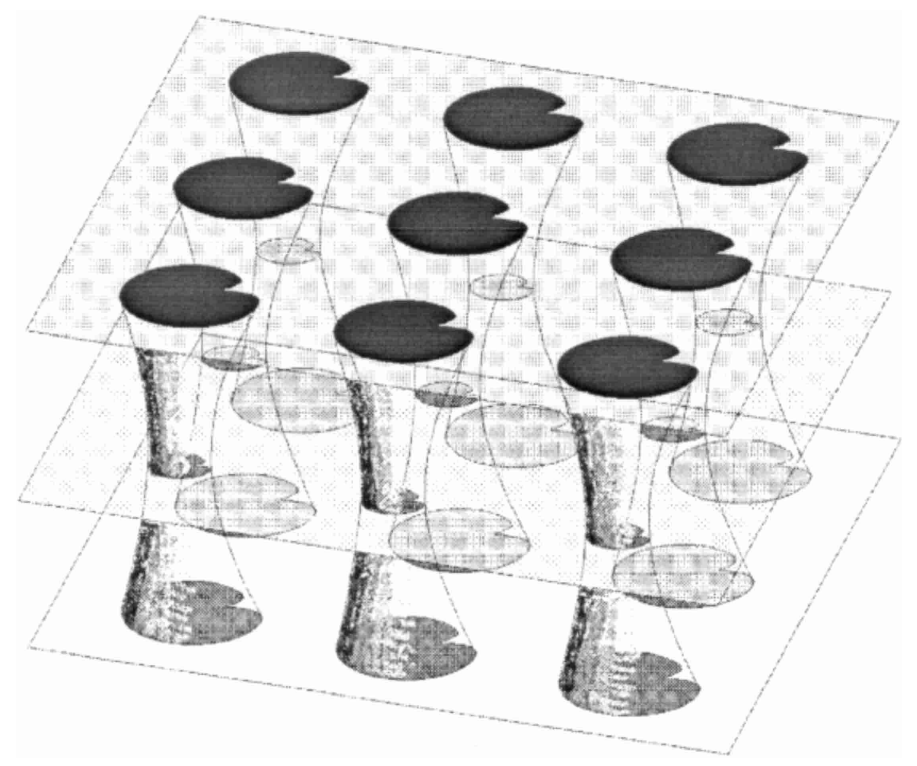

FIGURE 1. "Pulsing" structure.

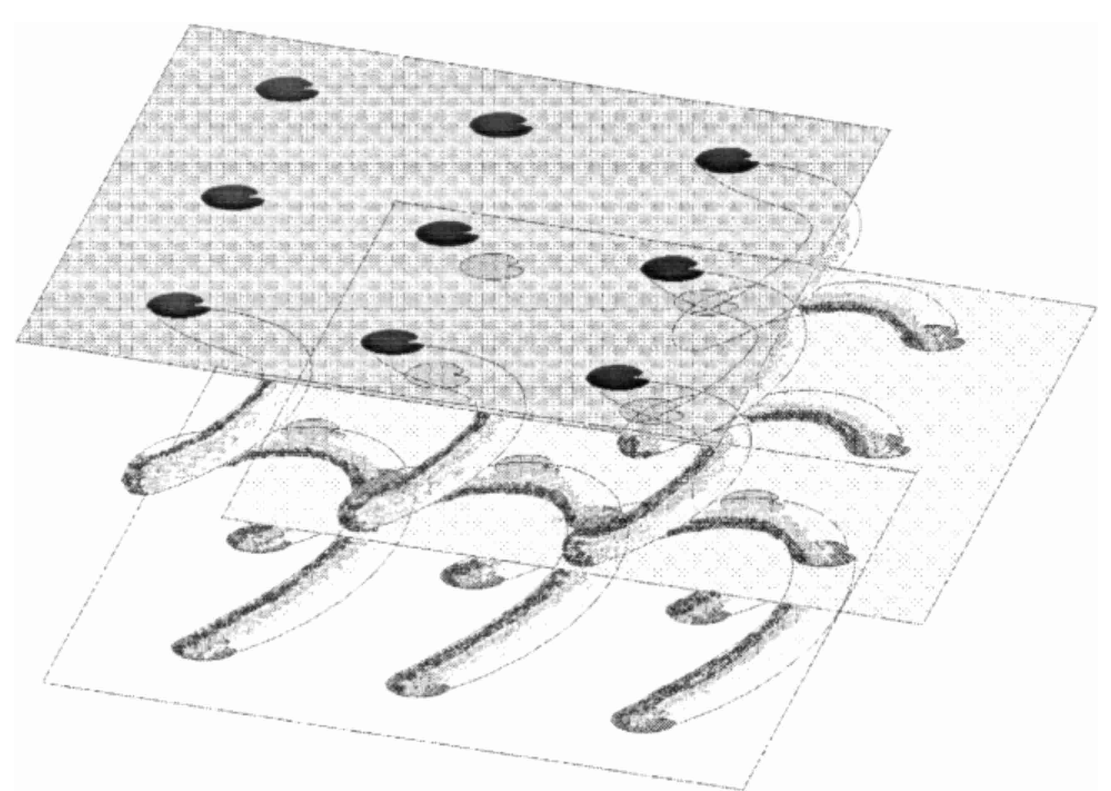

Figure 2. Helicoidal ("snakes-like") structure.

\section{JUSTIFICATION OF THE ASYMPTOTIC EXPANSION}

In this section we justify the asymptotic expansion of the solution $u^{\varepsilon}$ constructed in the previous section, and prove error estimates. 
Denote by $W^{\varepsilon}$ the sum of the first five terms of expansion (4.1), i.e.,

$$
\begin{aligned}
W^{\varepsilon}(x, t)= & u_{0}\left(x-\frac{\alpha}{\varepsilon} t, t\right)+\varepsilon\left\{\nabla_{x} u_{0}\left(x-\frac{\alpha}{\varepsilon} t, t\right) \chi\left(\frac{x}{\varepsilon}, \frac{t}{\varepsilon^{2}}\right)+u_{1}\left(x-\frac{\alpha}{\varepsilon} t, t\right)\right\} \\
& +\varepsilon^{2}\left\{\nabla_{x} \nabla_{x} u_{0}\left(x-\frac{\alpha}{\varepsilon} t, t\right) \xi\left(\frac{x}{\varepsilon}, \frac{t}{\varepsilon^{2}}\right)+\nabla_{x} u_{0}\left(x-\frac{\alpha}{\varepsilon} t, t\right) \chi\left(\frac{x}{\varepsilon}, \frac{t}{\varepsilon^{2}}\right)\right\} .
\end{aligned}
$$

Theorem 5.1. Suppose that the data of problem (1.3) are sufficiently smooth. Then the following estimates hold:

$$
\left\|u^{\varepsilon}-W^{\varepsilon}\right\|_{L^{2}\left(0, T ; H^{1}\left(G^{\varepsilon}(\cdot)\right)\right)} \leq C \varepsilon^{2}\left(\|f\|_{L^{2}\left(0, T ; H^{5}\left(\mathbb{R}^{n}\right)\right)}+\|\varphi\|_{H^{5}\left(\mathbb{R}^{n}\right)}\right)
$$

and

$$
\left\|u^{\varepsilon}-W^{\varepsilon}\right\|_{\left.C\left(Q_{T}^{\varepsilon}\right)\right)} \leq C \varepsilon^{2}\left(\|f\|_{L^{\infty}\left(0, T ; W^{5, \infty}\left(\mathbb{R}^{n}\right)\right)}+\|\varphi\|_{W^{5, \infty}\left(\mathbb{R}^{n}\right)}\right) .
$$

Proof. Substituting the difference $u^{\varepsilon}-W^{\varepsilon}$ in (1.3), and recalling (4.7) lead to an equation of the form

$$
\begin{aligned}
\frac{\partial\left[u^{\varepsilon}(x, t)-W^{\varepsilon}(x, t)\right]}{\partial t}-\Delta\left[u^{\varepsilon}(x, t)-W^{\varepsilon}(x, t)\right]= & \varepsilon^{2} \sum_{i} \beta_{1, i}\left(\frac{x}{\varepsilon}, \frac{t}{\varepsilon^{2}}\right) v_{1, i}(x, t) \\
& +\varepsilon^{3} \sum_{i} \beta_{2, i}\left(\frac{x}{\varepsilon}, \frac{t}{\varepsilon^{2}}\right) v_{2, i}(x, t) \quad \text { in } Q_{T}^{\varepsilon}
\end{aligned}
$$

with the following boundary condition on $\partial Q_{T}^{\varepsilon}$ :

$$
\left.\frac{\partial\left(u^{\varepsilon}-W^{\varepsilon}\right)}{\partial n^{\varepsilon}}\right|_{\partial Q_{T}^{\varepsilon}}=\varepsilon^{3} \sum_{i} \beta_{3, i}\left(\frac{x}{\varepsilon}, \frac{t}{\varepsilon^{2}}\right) \cdot v_{3, i}(x, t) .
$$

The functions $\beta_{l, i}=\beta_{l, i}(z, \tau)$ are smooth and periodic in $z$ and $\tau$; the functions $v_{1, i}$ and $v_{2, i}$ are linear combinations of fourth and fifth order derivatives of $u_{0}$, third and fourth of $u_{1}$ and second and third derivatives of $f$, all the derivatives are taken in space variables. Similarly, the function $v_{3, i}$ is a linear combination of third and fourth order derivatives of $u_{0}$ and second and third derivatives of $u_{1}$.

Due to standard parabolic estimates, the function $u_{0}=u_{0}(x, t)$ belongs to the space $L^{2}\left(0, T ; H^{5}\left(\mathbb{R}^{n}\right)\right)$, and moreover, one has the following inequality:

$$
\left\|u_{0}\right\|_{L^{2}\left(0, T ; H^{5}\left(\mathbb{R}^{n}\right)\right)} \leq C\left(\|f\|_{L^{2}\left(0, T ; H^{5}\left(\mathbb{R}^{n}\right)\right)}+\|\varphi\|_{H^{5}\left(\mathbb{R}^{n}\right)}\right),
$$

where $C$ is a constant independent of $u_{0}, f$ and $\varphi$. The same is true for $u_{1}$ too. Consequently, estimates (5.1) and (5.2) follow immediately from Proposition 3.1.

Now, denote by $Z^{\varepsilon}$ the sum of the first two terms of the expansion (4.1), i.e.,

$$
Z^{\varepsilon}(x, t)=u_{0}\left(x-\frac{\alpha}{\varepsilon} t, t\right)+\varepsilon\left\{\nabla_{x} u_{0}\left(x-\frac{\alpha}{\varepsilon} t, t\right) \chi\left(\frac{x}{\varepsilon}, \frac{t}{\varepsilon^{2}}\right)+u_{1}\left(x-\frac{\alpha}{\varepsilon} t, t\right)\right\} .
$$

Then, the following theorem holds:

Theorem 5.2. For any $f \in L^{2}\left(0, T ; L^{2}\left(\mathbb{R}^{n}\right)\right)$ and $\varphi \in H^{1}\left(\mathbb{R}^{n}\right)$, one has

$$
\lim _{\varepsilon \rightarrow 0}\left\|u^{\varepsilon}-Z^{\varepsilon}\right\|_{L^{2}\left(0, T ; H^{1}\left(\mathbb{R}^{n}\right)\right)}=0 .
$$


For $f \in L^{2}\left(0, T ; L^{2}\left(\mathbb{R}^{n}\right)\right)$ and $\varphi \in L^{2}\left(\mathbb{R}^{n}\right)$ we have

$$
\lim _{\varepsilon \rightarrow 0}\left\|u^{\varepsilon}-u_{0}\right\|_{L^{2}\left(0, T ; L^{2}\left(\mathbb{R}^{n}\right)\right)}=0 .
$$

Proof. In both cases, in order to prove the result it is sufficient to approach $f$ and $\varphi$ by smooth functions.

Let us prove convergence (5.3). Suppose that the sequences $\left\{f^{n}\right\} \subset C^{\infty}\left(0, T ; C_{0}^{\infty}\left(\mathbb{R}^{n}\right)\right)$ and $\left\{\varphi^{n}\right\} \subset C_{0}^{\infty}\left(\mathbb{R}^{n}\right)$, are such that

$$
\left\{\begin{array}{l}
\lim _{n \rightarrow \infty}\left\|f-f^{n}\right\|_{L^{2}\left(0, T ; L^{2}\left(\mathbb{R}^{n}\right)\right)}=0 \\
\lim _{n \rightarrow \infty}\left\|\varphi-\varphi^{n}\right\|_{H^{1}\left(\mathbb{R}^{n}\right)}=0 .
\end{array}\right.
$$

Denote by $u_{n}^{\varepsilon}$ the solution of problem (1.3) with the data $f^{n}$ and $\varphi^{n}$, and by $U_{n, 1}^{\varepsilon}$ the sum of the first four terms of the corresponding asymptotic expansion. Then we have

$$
\begin{aligned}
\left\|u^{\varepsilon}-Z^{\varepsilon}\right\|_{L^{2}\left(0, T ; H^{1}\left(\mathbb{R}^{n}\right)\right)} \leq & \left\|u^{\varepsilon}-u_{n}^{\varepsilon}\right\|_{L^{2}\left(0, T ; H^{1}\left(\mathbb{R}^{n}\right)\right)}+\left\|u_{n}^{\varepsilon}-U_{n, 1}^{\varepsilon}\right\|_{L^{2}\left(0, T ; H^{1}\left(\mathbb{R}^{n}\right)\right)} \\
& +\left\|U_{n, 1}^{\varepsilon}-Z^{\varepsilon}\right\|_{L^{2}\left(0, T ; H^{1}\left(\mathbb{R}^{n}\right)\right)} .
\end{aligned}
$$

By (5.5) and Proposition 3.1 the first term on the right-hand side converges to 0 , as $n \rightarrow \infty$, uniformly in $\varepsilon$. Similar convergence of the last term is a simple consequence of standard parabolic estimates. Finally, the middle term converges to 0 , as $\varepsilon \rightarrow 0$, by Theorem 5.1. Hence (5.2) is proved.

Finally, convergence (5.4) is obtained by the same arguments.

Remark 5.3. In the case of smooth data, one can construct higher order terms in the asymptotic expansion (4.1) and obtain estimates in more regular functional spaces.

\section{An EXAMPLE}

In order to show that in general $\alpha \neq 0$, let us consider a particular case of problem (1.3), when the evolution of the holes in the rescaled coordinates $\tau=t / \varepsilon^{2}, y=x / \varepsilon, y \in \mathbb{R} 2$, is defined as follows:

$$
G_{t}= \begin{cases}G_{0}+t e_{1}, & 0 \leq t \leq T, \\ G_{t}=G_{0}+(T-t) e_{1}, & T \leq t \leq 2 T,\end{cases}
$$

where $e_{1}=(1,0)$ is the first coordinate unit vector, and $G_{t}$ is a $2 T$-periodic function. The shape of a crosssection of the structure and the shape of holes do not depend on time. They are shown in Figures 3 and 4.

Notice that each hole merely walks in time along a "zig-zag" line as shown in Figure 5.

We have two "free" parameters, $T$ and $L$, at our disposal, and want to determine them to get $\alpha \neq 0$. We begin by introducing a $2 T$-periodic function

$$
\gamma(t)= \begin{cases}t, & 0 \leq t \leq T, \\ T-t, & T \leq t \leq 2 T,\end{cases}
$$

and make the change of coordinates

$$
(x, t) \longrightarrow\left(x+\varepsilon \gamma\left(\frac{t}{\varepsilon^{2}}\right) e_{1}, T\right)
$$


HOMOGENIZATION IN PERFORATED DOMAINS WITH RAPIDLY PULSING PERFORATIONS

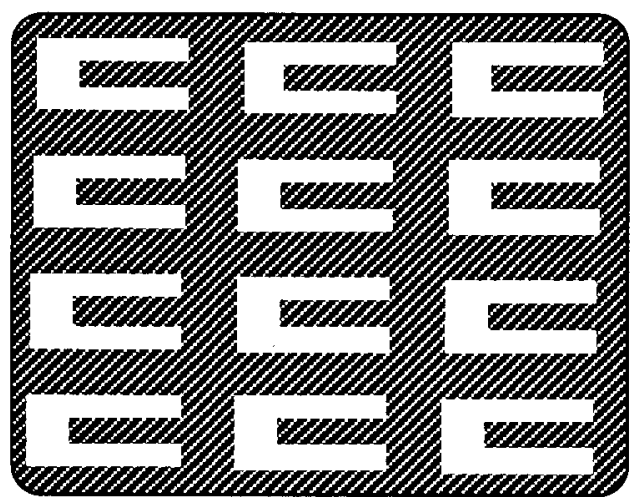

Figure 3. Shape of $G_{0}$ (set of white "holes").

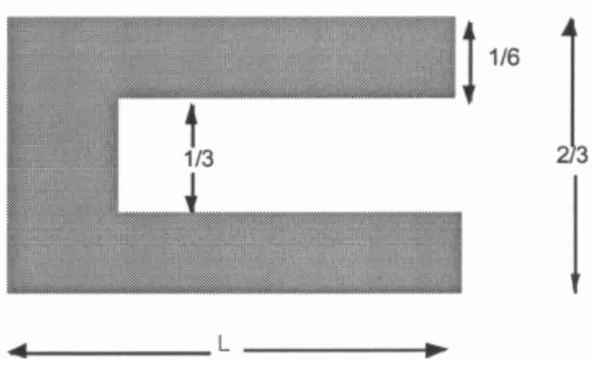

Figure 4. Shape of a hole.

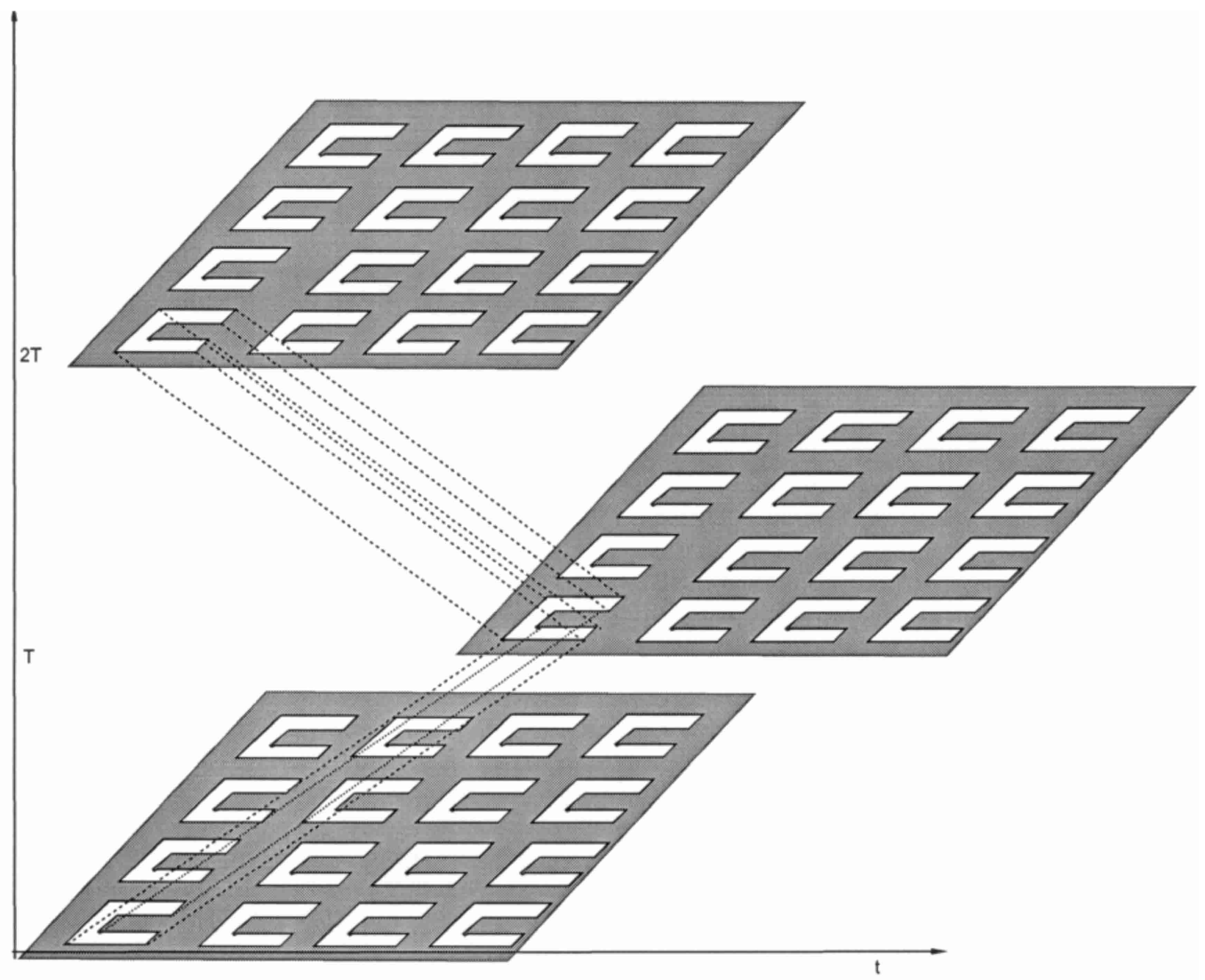

FiguRE 5. "Zig-zag" structure.

In the new coordinates (still denoted $(x, t)$ ), problem (1.3) (with $f \equiv 0$ ), has the form

$$
\left\{\begin{array}{l}
\frac{\partial u^{\varepsilon}}{\partial t}-\Delta u^{\varepsilon}-\frac{1}{\varepsilon} \gamma^{\prime}\left(\frac{t}{\varepsilon^{2}}\right) \frac{\partial u^{\varepsilon}}{\partial x_{1}}=0 \quad \text { in } Q_{T}^{\varepsilon} \\
\frac{\partial u^{\varepsilon}}{\partial n^{\varepsilon}}=0 \quad \text { on } \partial G_{0}^{\varepsilon} \\
\left.u^{\varepsilon}\right|_{t=0}=g \quad \text { in } G_{0}^{\varepsilon},
\end{array}\right.
$$


where $G_{0}^{\varepsilon}=\varepsilon G_{0}$. Obviously, $\gamma \prime$ is a $2 T$-periodic function, and

$$
\gamma^{\prime}(t)= \begin{cases}1, & 0 \leq t \leq T \\ -1, & T \leq t \leq 2 T .\end{cases}
$$

Observe that in problem (6.2) the geometry of holes is independent of time, that is the shape and the position of the holes is now fixed. Thus, we have a "classical" homogenization problem with oscillating coefficients set in a perforated domain. However, the new operator has large oscillating coefficients.

Taking into account the change of variables (6.1), we immediately see that the homogenized problems corresponding to (1.3) (or (1.5)) and (6.2) coincide, so we will work with the latter one.

Let us now consider the following auxiliary "cell" problem whose unknown is $p=p(z, \tau)$ :

$$
\begin{cases}\frac{\partial p}{\partial \tau}+\Delta p-\gamma^{\prime}(\tau) \frac{\partial p}{\partial z_{1}}=0 & \text { in } G_{0} \\ \frac{\partial p}{\partial n}-\gamma^{\prime}(\tau) n_{1}(z) p=0 & \text { on } \partial G_{0}\end{cases}
$$

to be studied in the space of periodic in $z$ and $\tau$ functions. In the same way as in Section 3, one can prove that (6.3) has a unique (up to a multiplicative constant) solution and this solution is positive. Let us denote by $G_{0}^{\text {per }}$ the following set:

$$
G_{0}^{\mathrm{per}}=[0, L+1] \times[0,1] \cap G_{0} .
$$

It is easy to see that

$$
\int_{G_{0}^{\text {per }}} p(z, \tau) \mathrm{d} z=C
$$

where $C$ is a constant independent of $\tau$. We fix the choice of $p$ by imposing the normalization

$$
C=1 .
$$

Also, like in Section 3, one can show that for periodic functions $f=f(z, \tau)$ and $g=g(z, \tau)$, the system

$$
\left\{\begin{array}{l}
\frac{\partial v}{\partial \tau}-\Delta v-\gamma^{\prime}(\tau) \frac{\partial v}{\partial z_{1}}=f \quad \text { in } G_{0} \\
\frac{\partial v}{\partial n}=g \quad \text { on } \partial G_{0}
\end{array}\right.
$$

has a solution in the space of periodic function if and only if

$$
\int_{0}^{2 T} \mathrm{~d} \tau \int_{G_{0}^{\text {per }}} f(z, \tau) p(z, \tau) \mathrm{d} z+\int_{0}^{2 T} \mathrm{~d} \tau \int_{\partial G_{0}^{\text {per }}} g(z, \tau) p(z, \tau) \mathrm{d} s_{z}=0 .
$$

Furthermore, in the same way as in Section 4, we obtain uniform a priori estimates for the solution $u^{\varepsilon}$ of problem (6.2). Then, following the scheme of Section 5, we represent $u^{\varepsilon}$ in the form

$$
\begin{aligned}
u^{\varepsilon}(x, t) \sim u_{0}(x-\bar{\alpha} t, t)+\varepsilon\left\{\nabla_{x} u_{0}(x-\bar{\alpha} t, t) \chi\left(\frac{x}{\varepsilon}, \frac{t}{\varepsilon^{2}}\right)+u_{1}(\right. & x-\bar{\alpha} t, t)\} \\
& +\varepsilon^{2} \nabla_{x} \nabla_{x} u_{0}(x-\bar{\alpha} t, t) \xi\left(\frac{x}{\varepsilon}, \frac{t}{\varepsilon^{2}}\right)+\cdots
\end{aligned}
$$


Due to the symmetry of $G_{0}$ in $z_{2}$, the vector $\bar{\alpha}$ must be of the form $\bar{\alpha}=(\alpha, 0)$.

Substituting (6.6) in (6.2), and equating the power-like terms of $\varepsilon$, we obtain in particular, the following problem to determine $\chi^{1}=\chi^{1}(z, \tau)$ :

$$
\left\{\begin{array}{l}
\frac{\partial \chi^{1}}{\partial \tau}-\Delta \chi^{1}-\gamma^{\prime}(\tau) \frac{\partial \chi^{1}}{\partial z_{1}}=\alpha+\gamma^{\prime}(\tau) \quad \text { in } G_{0} \\
\frac{\partial \chi^{1}}{\partial n}=-n_{1} \quad \text { on } \partial G_{0} .
\end{array}\right.
$$

Thanks to the evident identities

$$
\begin{aligned}
& \int_{0}^{2 T} \mathrm{~d} \tau \int_{G_{0}^{\text {per }}} \gamma^{\prime}(\tau) p(z, \tau) \mathrm{d} z=\int_{0}^{2 T} \gamma^{\prime}(\tau) \mathrm{d} \tau=0 \\
& \int_{G_{0}^{\text {per }}} \alpha p(z, \tau) \mathrm{d} z=2 T \alpha
\end{aligned}
$$

the compatibility condition (6.5) for system (6.3) reads,

$$
2 T \alpha=\int_{0}^{2 T} \mathrm{~d} \tau \int_{\partial G_{0}^{\text {per }}} n_{1}(z) p(z, \tau) \mathrm{d} s_{z} .
$$

Our aim is now to select the parameters $L$ and $T$ so that $\alpha \neq 0$. To this end note first that on the interval [0,T], equation (6.3) takes the form

Moreover, one has

$$
\left\{\begin{array}{l}
\frac{\partial p}{\partial \tau}+\Delta p-\frac{\partial p}{\partial z_{1}}=0 \quad \text { in } G_{0}^{\mathrm{per}} \\
\frac{\partial p}{\partial n}-n_{1} p=0 \quad \text { on } \partial G_{0}^{\mathrm{per}}
\end{array}\right.
$$

$$
\int_{G_{0}} p(z, \tau) \mathrm{d} z=1, \quad\|p(\cdot, T)\|_{L^{2}\left(G_{0}\right)} \leq C,
$$

where the latter relation is a standard parabolic estimate. It is uniform in $T$.

We consider the behaviour of a solution $p(z, \tau)$ on the time interval $(0, T)$ for large $T$. The results from Section 3 imply the convergence of $p$, as $(t-T) \rightarrow-\infty$, at an exponential rate, towards the unique solution of the elliptic problem

$$
\left\{\begin{array}{l}
\Delta p^{1}-\frac{\partial p_{1}}{\partial z_{1}}=0 \quad \text { in } G_{0}^{\text {per }} \\
\frac{\partial p_{1}}{\partial n}-n_{1} p_{1}=0 \quad \text { on } \partial G_{0}^{\text {per }} \\
\int_{G_{0}^{\text {per }}} p_{1}(z) \mathrm{d} z=1
\end{array}\right.
$$

Therefore, the contribution of the set $G_{0}^{\mathrm{per}} \times[0, T]$ to the integral in (6.7), is given by

$$
\int_{0}^{T} \mathrm{~d} \tau \int_{\partial G_{0}^{\text {per }}} n_{1}(z) p(z, \tau) \mathrm{d} s_{z}=[1+o(1)] T \alpha_{1},
$$




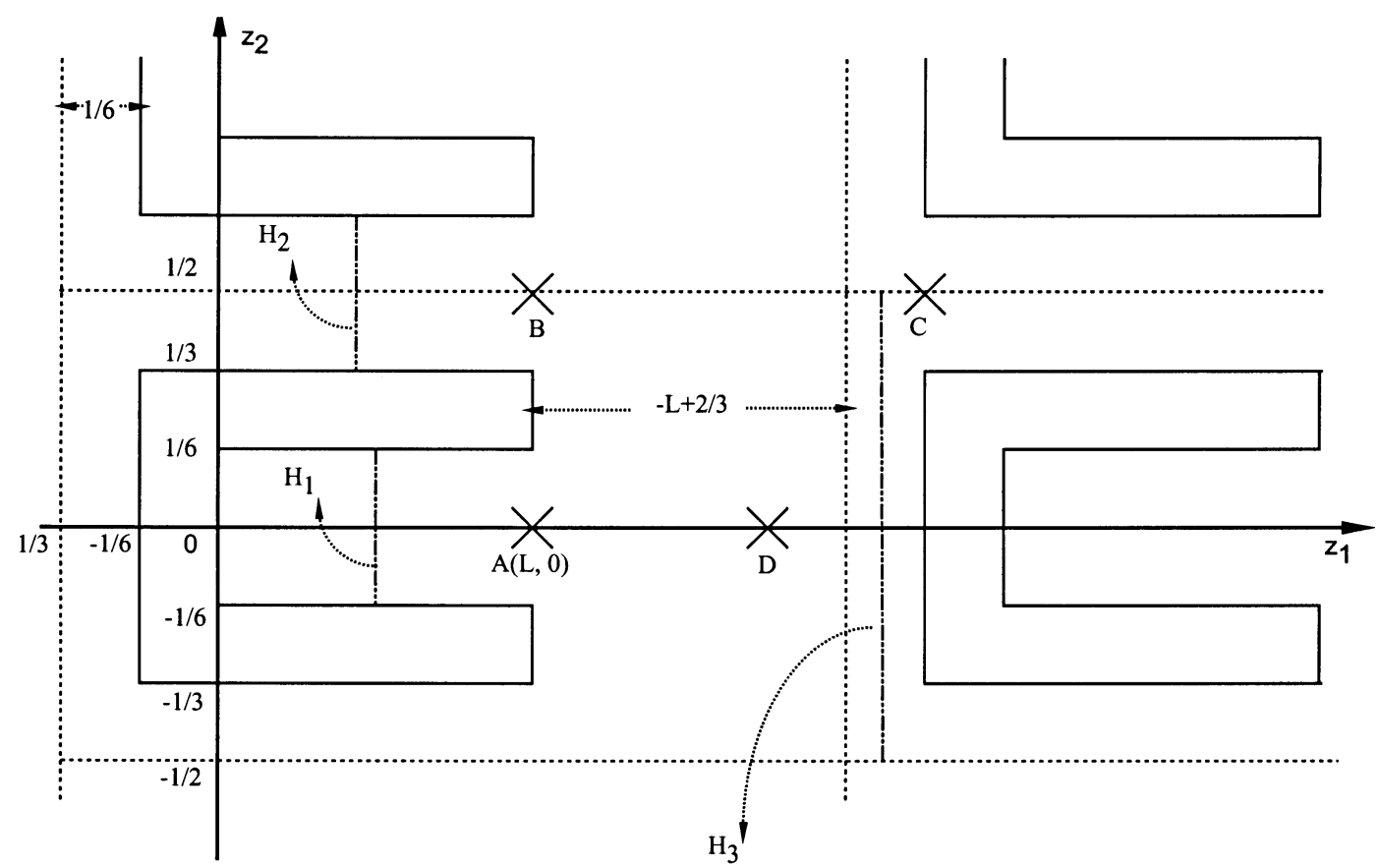

Figure 6. Cross-sections in $G_{0}$.

where

$$
\alpha_{1}=\int_{\partial G_{0}^{\text {per }}} n_{1}(z) p_{1}(z) \mathrm{d} s_{z}
$$

and $o(1)$ vanishes as $T \rightarrow \infty$.

Similarly,

$$
\int_{T}^{2 T} \mathrm{~d} \tau \int_{\partial G_{0}^{\text {per }}} n_{1}(z) p(z, \tau) \mathrm{d} s_{z}=[1+o(1)] T \alpha_{2}
$$

where

$$
\alpha_{2}=\int_{\partial G_{0}^{\text {per }}} n_{1}(z) p_{2}(z) \mathrm{d} s_{z}
$$

and $p_{2}=p_{2}(z)$ solves the problem

$$
\left\{\begin{array}{l}
\Delta p_{2}+\frac{\partial p_{2}}{\partial z_{1}}=0 \quad \text { in } G_{0}^{\text {per }} \\
\frac{\partial p_{2}}{\partial n}-n_{1} p_{2}=0 \quad \text { on } \partial G_{0}^{\text {per }} \\
\int_{G_{0}^{\text {per }}} p_{2}(z) \mathrm{d} z=1 .
\end{array}\right.
$$

It is now clear that it is sufficient to find $L>0$ such that $\alpha_{1} \neq-\alpha_{2}$. To this end, we study the behaviour of the integrals of $p_{1}$ and $p_{2}$ over the cross sections $H_{1}\left(z_{1}\right), H_{2}\left(z_{1}\right)$ and $H_{3}\left(z_{1}\right)$ (see Fig. 6 below). 
So, we have to compute the following integrals:

$$
\begin{aligned}
& I_{1}\left(z_{1}\right)=\int_{-1 / 6}^{+1 / 6} p_{1}(z) \mathrm{d} z_{2}, \quad 0 \leq z_{1} \leq L \\
& I_{2}\left(z_{1}\right)=\int_{1 / 3}^{2 / 3} p_{1}(z) \mathrm{d} \tau_{2}, \quad-\frac{1}{6} \leq z_{1} \leq L
\end{aligned}
$$

and (due to the periodicity)

$$
I_{3}\left(z_{1}\right)=\int_{0}^{1} p_{1}(z) \mathrm{d} \tau_{2}, \quad L \leq z_{1} \leq L+\frac{5}{6}
$$

Integrating (6.8) over each of the above cross sections, we get the equations

$$
\frac{\mathrm{d}^{2} I_{i}}{\mathrm{~d} z_{1}^{2}}-\frac{\mathrm{d} I_{i}}{\mathrm{~d} z_{1}}=0, \quad i=1,2,3
$$

with

whence

$$
\frac{\mathrm{d} I_{1}}{\mathrm{~d} \tau_{1}}(0)-I_{1}(0)=0
$$

$I_{1}\left(z_{1}\right)=C_{1} \mathrm{e}^{z_{1}}, \quad I_{2}\left(z_{1}\right)=C_{2} \mathrm{e}^{z_{1}}+d_{2}$,
where $C_{i}$ and $d_{i}$ are constants. By the Harnack inequality one has

$$
p_{1}(A) \leq c p_{1}(C), \quad p_{1}(A) \leq c p_{1}(B),
$$

with a constant $c$ independent of $L$. In view of the monotonicity of $I_{2}$, these bounds imply the estimate

$$
I_{2}\left(z_{1}\right) \geq c_{1} p_{1}(A), \quad-\frac{1}{6} \leq z_{1} \leq L .
$$

By integrating, we get

$$
c_{1} p_{1}(A) L \leq \int_{-\frac{1}{6}}^{L} I_{2}\left(z_{1}\right) \mathrm{d} z_{1} \leq 1
$$

where again we made use of the normalization condition (6.4). Consequently,

$$
p_{1}(A) \leq c_{2} \frac{1}{L} \text {. }
$$

Similarly, $p_{1}(D) \leq c p_{1}(A) \leq c_{3} \frac{1}{L}$, and hence

$$
I_{3}\left(z_{1}\right) \leq c_{4} \frac{1}{L}, \quad L \leq z_{1} \leq L+\frac{5}{6} .
$$

Finally, using the obvious bound $I_{1}(0) \leq c_{5} \frac{\mathrm{e}^{-L}}{L}$, we deduce the estimate

$$
\left|\alpha_{1}\right|=\left|\int_{\partial G_{0}^{\text {per }}} n_{1}(z) p_{1}(z) \mathrm{d} s_{z}\right| \leq I_{1}(0)+I_{3}(L)+I_{3}\left(L+\frac{5}{6}\right) \leq c_{6} \frac{1}{L} .
$$


In the same way, defining

$$
\begin{aligned}
& \mathcal{I}_{1}\left(z_{1}\right)=\int_{-1 / 6}^{+1 / 6} p_{2}(z) \mathrm{d} z_{2}, \quad 0 \leq z_{1} \leq L \\
& \mathcal{I}_{2}\left(z_{1}\right)=\int_{1 / 3}^{2 / 3} p_{2}(z) \mathrm{d} z_{2}, \quad-\frac{1}{6} \leq z_{1} \leq L \\
& \mathcal{I}_{3}\left(z_{1}\right)=\int_{0}^{1} p_{2}(z) \mathrm{d} z_{2}, \quad L \leq z_{1} \leq L+\frac{5}{6},
\end{aligned}
$$

we derive the following equations:

with

$$
\frac{\mathrm{d}^{2} \mathcal{I}_{i}}{\mathrm{~d} z_{1}^{2}}+\frac{\mathrm{d} \mathcal{I}_{i}}{\mathrm{~d} z_{1}}=0, \quad i=1,2,3
$$

This yields

$$
\frac{\mathrm{d} \mathcal{I}_{1}}{\mathrm{~d} z_{1}}(0)+\mathcal{I}_{1}(0)=0
$$

$$
\mathcal{I}_{1}\left(z_{1}\right)=\bar{C}_{1} \mathrm{e}^{-z_{1}}, \quad \mathcal{I}_{2}\left(z_{1}\right)=\bar{C}_{2} \mathrm{e}^{-z_{1}}+\bar{d}_{2}, \quad \mathcal{I}_{3}\left(z_{1}\right)=\bar{C}_{3} \mathrm{e}^{-z_{1}}+\bar{d}_{3} .
$$

In particular,

$$
p_{2}(A) \leq c_{7} \bar{C}_{1} \mathrm{e}^{-L},
$$

and by the Harnack inequality,

$$
p_{2}(A)+p_{2}(B)+p_{2}(D) \leq c_{8} \bar{C}_{1} \mathrm{e}^{-L} .
$$

The last inequality, after straightforward computations, leads to

$$
\int_{0}^{L} \mathcal{I}_{1}\left(z_{1}\right) \mathrm{d} z_{1} \leq \bar{C}_{1}(1-L), \quad \int_{-1 / 6}^{L} \mathcal{I}_{2}\left(z_{1}\right) \mathrm{d} z_{1} \leq c_{9} \bar{C}_{1} L \mathrm{e}^{-L}, \quad \int_{L}^{L+5 / 6} \mathcal{I}_{3}\left(z_{1}\right) \mathrm{d} z_{1} \leq c_{10} \bar{C}_{1} \mathrm{e}^{-L} .
$$

The normalization condition (6.4) implies that the sum of the three integrals above is equal to 1 , so from (6.10) we have that

$$
\bar{C}_{1}\left(1-\mathrm{e}^{-L}+c_{9} L \mathrm{e}^{-L}+c_{10} \mathrm{e}^{-L}\right) \geq 1 .
$$

Consequently, for $L$ sufficiently large

On the other hand,

$$
\bar{C}_{1} \geq \frac{1}{2} .
$$

$$
\bar{C}_{1} \leq 2,
$$

and we have

$$
\int_{-1 / 6}^{+1 / 6} p_{2}\left(0, z_{2}\right) \mathrm{d} z_{2}=\mathcal{I}_{1}(0) \geq \frac{1}{2}
$$

and also

$$
\int_{-1 / 3}^{-1 / 6} p_{2}\left(L, z_{2}\right) \mathrm{d} z_{2}+\int_{1 / 6}^{1 / 3} p_{2}\left(L, z_{2}\right) \mathrm{d} z_{2}+\int_{-1 / 3}^{1 / 3} p_{2}\left(L+\frac{5}{6}, z_{2}\right) \mathrm{d} z_{2} \leq \mathcal{I}_{3}(L)+\mathcal{I}_{3}\left(L+\frac{5}{6}\right) \leq 2 c_{10} \mathrm{e}^{-L} .
$$


The last two inequalities imply

$$
\alpha_{2}=\int_{\partial G_{0}^{\text {per }}} n_{1}(z) p_{2}(z) \mathrm{d} s_{z} \geq \frac{1}{4},
$$

for $L$ sufficiently large. Now, combining this relation with (6.9), we conclude that in this case,

$$
\alpha_{1}+\alpha_{2} \neq 0
$$

\section{REFERENCES}

[1] N.S. Bakhvalov, Averaging of partial differential equations with rapidly oscillating coefficients. Soviet Math. Dokl. 16 (1975).

[2] A. Bensoussan, J.L. Lions and G. Papanicolaou, Asymptotic analysis for periodic structures. North-Holland (1978).

[3] F. Campillo, M.L. Kleptsyna and A.L. Piatnitski, Homogenization of random parabolic operators with large potential. Stochastic Process. Appl. 93 (2001) 57-85.

[4] D. Cioranescu and J. Saint Jean Paulin, Homogenization in open sets with holes. J. Math. Anal. Appl. 71 (1979) $590-607$.

[5] D. Cioranescu and J. Saint Jean Paulin, Homogenization of reticulated structures. Springer-Verlag (1999).

[6] P. Donato and A. Nabil, Homogenization and correctors for the heat equation in perforated domains. Ricerche di Mat. L (2001) 115-144.

[7] A. Friedman, Partial differential equations of parabolic type. Prentice-Hall, Englewood Cliffs (1964).

[8] U. Hornung, Homogenization and porous media. Springer-Verlag, IAM 6 (1997).

[9] V.V. Jikov, S.M. Kozlov and O.A. Oleinik, Homogenization of differential operators and integral functionals. Springer-Verlag (1994).

[10] M.L. Kleptsyna and A.L. Piatnitski, Homogenization of random parabolic operators. Gakuto International Series. Math. Sci. Appl. 9 (1997), Homogenization and Appl. to Material Sciences, 241-255.

[11] M.L. Kleptsyna and A.L. Piatnitski, Averaging of non selfadjoint parabolic equations with random evolution (dynamics), Preprint INRIA. J. Funct. Anal. (submitted).

[12] M.A. Krasnosel'skii, E.A. Lifshits and E.A. Sobolev, Positive linear systems. The method of positive linear operators. Heldermann Verlag, Sigma Ser. Appl. Math. 5 (1989)

[13] A.L. Piatnitsky, Parabolic equations with rapidly oscillating coefficients (In Russian). English transl. Moscow Univ. Math. Bull. 3 (1980) 33-39. 